University of Nebraska - Lincoln

DigitalCommons@University of Nebraska - Lincoln

October 1981

\title{
Piezomodulation spectroscopy of molecular crystals. III. The first singlet system of anthracene
}

\author{
J. Merski \\ University of Nebraska - Lincoln \\ Craig J. Eckhardt \\ University of Nebraska - Lincoln, ceckhardt1@unl.edu
}

Follow this and additional works at: https://digitalcommons.unl.edu/chemistryeckhardt

Part of the Chemistry Commons

Merski, J. and Eckhardt, Craig J., "Piezomodulation spectroscopy of molecular crystals. III. The first singlet system of anthracene" (1981). Craig J. Eckhardt Publications. 34.

https://digitalcommons.unl.edu/chemistryeckhardt/34

This Article is brought to you for free and open access by the Published Research - Department of Chemistry at DigitalCommons@University of Nebraska - Lincoln. It has been accepted for inclusion in Craig J. Eckhardt Publications by an authorized administrator of DigitalCommons@University of Nebraska - Lincoln. 


\title{
Piezomodulation spectroscopy of molecular crystals. ${ }^{\text {a) }}$ III. The first singlet system of anthracene ${ }^{\text {b) }}$
}

\author{
J. Merskic) and C. J. Eckhardt ${ }^{d)}$ \\ Department of Chemistry, University of Nebraska, Lincoln, Nebraska 68588 \\ (Received 9 October 1980; accepted 3 March 1981)
}

\begin{abstract}
The polarized piezoreflection spectra of the first singlet system of anthracene and their Kramers-Kronig transforms are reported. Exciton energy strain shifts are reported for the $b$-polarized and for the $a c$-polarized factor group components. These values are shown to be in good agreement with related experimental data and with theoretical calculations. The piezomodulation spectra significantly enhance structure and are also sensitive to the extent of crystal coupling. Deviations in the measured spectra are observed in the $a$-polarized spectra from that calculated for the logarithmic derivative. These variances are attributed to coupling with the second singlet of anthracene or the presence of the ' $L_{b}$ transition.
\end{abstract}

\section{INTRODUCTION}

In the application of piezomodulation spectroscopy to the study of molecular crystals it is important that it be applied to a system which is relatively well understood. The optical spectroscopic investigations of the first singlet system in crystalline anthracene, mainly from the (001) face, are perhaps the most extensive of those in the literature of the spectroscopy of molecular crystals. Both the experimental and theoretical characterization of its lowest singlet vibronic exciton states establishes anthracene as an ideal model system. Hence, the diagnostic potential of piezomodulation techniques in probing molecular exciton states may be evaluated with some degree of certainty. Furthermore, the vibronic coupling among polariton states derived from the ${ }^{1} B_{2 u}-{ }^{1} A_{1}$ transition is weak to intermediate in strength. Consequently, exciton interactions in anthracene are more significant than those occuring in the PMDA-A system discussed in paper $\mathrm{II}$, but not as potent as those associated with the strongly coupled dye crystal systems which will be studied in the following paper.

Although a relatively recent review of exciton states in anthracene has appeared, ${ }^{1}$ it is useful to examine some pertinent developments which will become useful in later arguments. Study of the specular reflection spectra and their Kramers-Kronig transformed absorption spectra have provided information from not only the (001) face but also from the other naturally occuring faces of anthracene. ${ }^{2,3}$ In Table I are presented the factor group splittings for the singlet vibronic exciton states, obtained by calculation and measurement. As a result of these and other studies of anthracene, the $(0,0)$ factor group splitting is now generally accepted to be $200 \pm 10 \mathrm{~cm}^{-1}$ with a total factor group splitting of approximately $375 \mathrm{~cm}^{-1}$. The low temperature reflectivity data indicate that the total splitting increases by

\footnotetext{
2) Research supported by the Solid State Chemistry Program, Division of Materials Research of the National Science Foundation (DMR-79-08-759).

b) Part I of the series is J. Merski and C. J. Eckhardt, J. Chem. Phys. 75, 3691 (1981); part II is 75, 3705 (1981).

") Part of a dissertation submitted to the University of Nebraska in partial requirement for the $\mathrm{Ph} . \mathrm{D}$. degree.

John Simon Guggenheim Fellow, 1979-80.
}

more than $75 \mathrm{~cm}^{-1}$ relative to that observed at ambient temperature.

The theory of exciton states in anthracene has been developed to the point where the principal features of the first singlet exciton spectra are reasonably well understood. The weak coupling limit of dipole theory provides an incomplete description of the system, however, and higher multipole interactions as well as interaction with the long axis polarized ${ }^{1} B_{1 u}-{ }^{1} A_{1}$ second singlet transition must be included. Calculations employing high and low temperature lattice parameters have indicated that the increase of factor group splitting due to thermal contraction should be $68 \mathrm{~cm}^{-1}$. Thus, results obtained from the appropriately modified dipole theory provide quite good agreement between theory and experiment.

The relevant $x$-ray crystallographic data and the pressure dependence of the lattice parameters together with the experimental procedures and free molecule spectroscopic parameters which are germane to considerations of this section are detailed in Sec. II. In Sec. III, the primary experimental data $R$ and $\Delta R / R$ are introduced along with the corresponding Seraphin coefficients $\alpha$ and $\beta$ and the calculated logarithmic photon energy derivatives $(1 / R)(d R / d \bar{\nu})$. The differential Kramers Kronig transformed quantities $\Delta \theta$ and $\Delta \bar{\epsilon}$ are presented in Sec. IV. The $\Delta R / R$ and $\Delta \tilde{\epsilon}$ spectral profiles are compared with the unmodulated $R$ and Kramers-Kronig transformation data ${ }^{2}$ and the high resolution $2 \mathrm{~K}$ reflection spectra. ${ }^{3,4}$ The correspondence between the piezoreflection spectra and the numerically calculated log-

TABLE I. Experimental and theoretical factor group splittings for the (001) face of anthracene.

\begin{tabular}{|c|c|c|c|c|}
\hline \multirow{3}{*}{$\begin{array}{l}\text { Vibronic } \\
\text { component }\end{array}$} & & & \multicolumn{2}{|c|}{ Theoretical $\left(\mathrm{cm}^{-1}\right)$} \\
\hline & \multicolumn{2}{|c|}{ Experimental $\left(\mathrm{cm}^{-1}\right)$} & \multirow{2}{*}{$\begin{array}{l}\text { Silbey, } \\
\text { Jortner, } \\
\text { and Ricec }\end{array}$} & \multirow{2}{*}{$\begin{array}{l}\text { Schlosser } \\
\text { and } \\
\text { Philpott }^{\complement}\end{array}$} \\
\hline & $298 \mathrm{~K}^{a}$ & $77 \mathrm{~K}^{\mathrm{b}}$ & & \\
\hline$(0,0)$ & $190(210)^{b}$ & 266 & 207 & 235 \\
\hline$(0,1)$ & 110 & 110 & 102 & 99 \\
\hline$(0,2)$ & 60 & 50 & 54 & 53 \\
\hline
\end{tabular}

Beference $3 . \quad$ Reference 21. 
arithmic derivatives $(1 / R)(d R / d \bar{\nu})$ is also examined. Section $\mathrm{V}$ involves a discussion of the strain sensitivi ties of the vibronic exciton states, where the differences in deformation potentials between ground and excited crystal states determined from the piezospectroscopic data are presented.

Comparison with values from high pressure studies and from low temperature work and the values reported from this research is made. The strain shift results for the anthracene first singlet in the PMDA-A pi complex crystal and those for the singlet in pure anthracene are also compared. Finally, the measured strain shifts are compared to those calculated using the dispersion and exciton resonance energy derivatives given by Schipper. 5

\section{EXPERIMENTAL}

\section{A. Crystal preparation and physical properties}

Commercially available blue-violet fluorescence grade anthracene was used without further purification except when plates cleaved from a Bridgman-grown boule were used. Thin plate-like crystals $[(001)$ plate face] were obtained from a nearly saturated solution of anthracene in methyl ethyl ketone which was purified by distillation over molecular sieves. The anthracene crystals were stored in the dark under an atmosphere of dry nitrogen. The platelets selected for piezore flection studies were estimated to be approximately 100 $\mu$ thick. Piezoreflection spectra were obtained from the (001) face at $298 \mathrm{~K}$ for incident light polarization directions coincident with either the a or b crystal axis. Experimental details are to be published elsewhere.

The anthracene structure, lattice parameters, and unit cell volumes at 290 and $95 \mathrm{~K}$ are reported by Mason $^{6}$ from which are calculated the fractional change in the unit cell volume $\Delta v / v_{0}$ of $-3.57 \times 10^{-2}$ and fractional change in the surface area of the (001) face of $-1.98 \times 10^{-2}$. This fractional change in volume due to thermal contraction would require the application of an equivalent hydrostatic pressure of approximately 5 kbar. The latter was calculated using the bulk isothermal compressibility data for anthracene from Bridgman? Comparable estimates for the equivalent pressure of 3.8 and $3.4 \mathrm{kbar}$ may be obtained using the compressibility data of Samara and Drickamer, and Rice and Jortner, ${ }^{9}$ respectively. Recently, the pressure dependence of the anthracene lattice parameters has been measured by Elnahwy et al. ${ }^{10}$ in the pressure range from 0 to $5.4 \mathrm{kbar}$ employing neutron diffraction techniques. These workers report the following pressure dependencies for the $a, b, c$, and $\beta$ lattice parameters:

$$
\begin{aligned}
& \frac{\partial a}{\partial P}=-0.042 \pm 0.002 \AA / \mathrm{kbar}, \\
& \frac{\partial b}{\partial P}=-0.0172 \pm 0.005 \AA / \mathrm{kbar}, \\
& \frac{\partial c}{\partial P}=-0.031 \pm 0.003 \AA / \mathrm{kbar}, \\
& \frac{\partial \beta}{\partial P}=0.08^{\circ} \pm 0.01^{\circ} / \mathrm{kbar} .
\end{aligned}
$$

From these, Elnahwy et al. derived an improved set of elastic constants. These more recent data indicate that a hydrostatic pressure of approximately $3.1 \mathrm{kbar}$ would be required to induce a volume change of the crystal equivalent to the thermal contraction found from 290 to $95 \mathrm{~K}$. The equivalent pressures discussed herein are only approximations, regardless of the quality of the crystal compressibility data, because the anisotropy of the thermal expansion coefficients is similar, but not identical, to that of the elastic constants or linear compressibilities. With this qualification in mind, one may extract some comparisons among the strain (or pressure) shift data obtained for the first singlet system of anthracene from piezomodulation studies, high pressure optical spectroscopy, and temperature-dependent crystal spectroscopy experiments. The values of Elnahwy et al. are used throughout this study.

The first singlet vibronic exciton states in anthracene arise from the short axis, $\hat{\mathrm{M}}_{\mathrm{A}}$ polarized, ${ }^{1} B_{2 u}-{ }^{1} A_{1 \varepsilon}$ transition. The squared projections of $\hat{\mathbf{M}}_{\mathbf{A}}$ onto the a and $b$ directions of the (001) face yield a theoretical oriented gas $b / a$ polarization ratio of 7.94 . In contrast to the latter, the experimental polarization ratios for the $(0,0),(0,1),(0,2)$, and $(0,3)$ vibronic transitions are reported as $4.7,3.2,2.8$, and 2.2 , respectively. These values clearly indicate the significance of interactions between the first singlet system and higher energy excitations such as the intense long axis, $\hat{L}_{\boldsymbol{A}}$ polar ized, ${ }^{1} B_{1 u}-{ }^{1} A_{1 g}$ transition. The strain modulation of such interactions would be manifested in the piezomodulation spectra of the first singlet system. The symmetry preserving components of the crystal strain perturb the existing degenerate and nondegenerate level interactions whereas the symmetry breaking strain components induce second and higher order couplings which do not exist in the unstrained crystal.

\section{B. Molecular spectroscopy information and site shifts}

It is necessary to assess the magnitude of the vaporto-crystal or site shift of the excitation energy for use in the parametrization of the dispersion derivative lattice sums which facilitate calculation of the straininduced change in the site shift. The difference in dispersion energies $D$ between the ground and excited states in the crystal given by Schipper is $-1980 \mathrm{~cm}^{-1}$. The calculated exciton strain shifts will use this value for $D$. The "correct" value to use for the site shift is not entirely certain. Philpott ${ }^{11}$ uses a value for $D$ of $-1688 \mathrm{~cm}^{-1}$ in his exciton calculations for the first singlet system of anthracene. This site shift represents the mean value of the vapor-to-mixed crystal excitation energy shifts. In contrast, Rice and Jortner ${ }^{9}$ select a value of $-1860 \mathrm{~cm}^{-1}$ for $D$ obtained from a comparison of the $(0,3)$ and $(0,4)$ vibronic transitions energies observed in the pure crystal absorption spectrum with the corresponding excitation energies in the vapor phase spectrum. This procedure takes cognizance of the small Franck-Condon factors for the $(0,3)$ and $(0,4)$ vibronic transitions which, in turn, implies negligible contributions to the crystal excitation energies due to the exciton interactions. Hence, one may advance the argument that the high values for the site shift of either 
-1980 or $-1860 \mathrm{~cm}^{-1}$ are somewhat more appropriate than $-1688 \mathrm{~cm}^{-1}$. The accuracy of these values is limited, however, because the higher energy vibronic transitions give rise to rather weak and diffuse absorption bands and consequently the error in the determination of the transition energies is sizeable. However, noting the approximations inherent in the dispersion derivative calculations (only $a$ and $b$ strains are explicitly considered), these differences in the values for the site shift are insignificant.

\section{Piezospectroscopic data}

The piezoreflection spectra presented herein constitute a representative sampling of experimental data acquired from four different crystals. Each plotted a or b polarized $\Delta R / R$ spectrum represents a numerical average of four spectra which were assigned equal statistical weights. During the course of the modulated piezoreflection studies of anthracene more than 10 crystals were investigated and the variances in the ener gies of the principal features contained therein were within spectral resolution. The variances in the magnitudes of $\Delta R / R$ among the individual piezoreflection spectra constituting the average were found to be approximately $\pm 5 \%$. The low and high resolution $\Delta R / R$ spectra were obtained by operating the Spex 1400-II monochromator in the double dispersion mode with bandpasses of 60 and $10 \mathrm{~cm}^{-1}$ (third order), respectively.

Modulated interference effects were observed in the $\Delta R / R$ spectra of anthracene to the red of the $(0,0)$ vibronic transition due to light reflected from the back

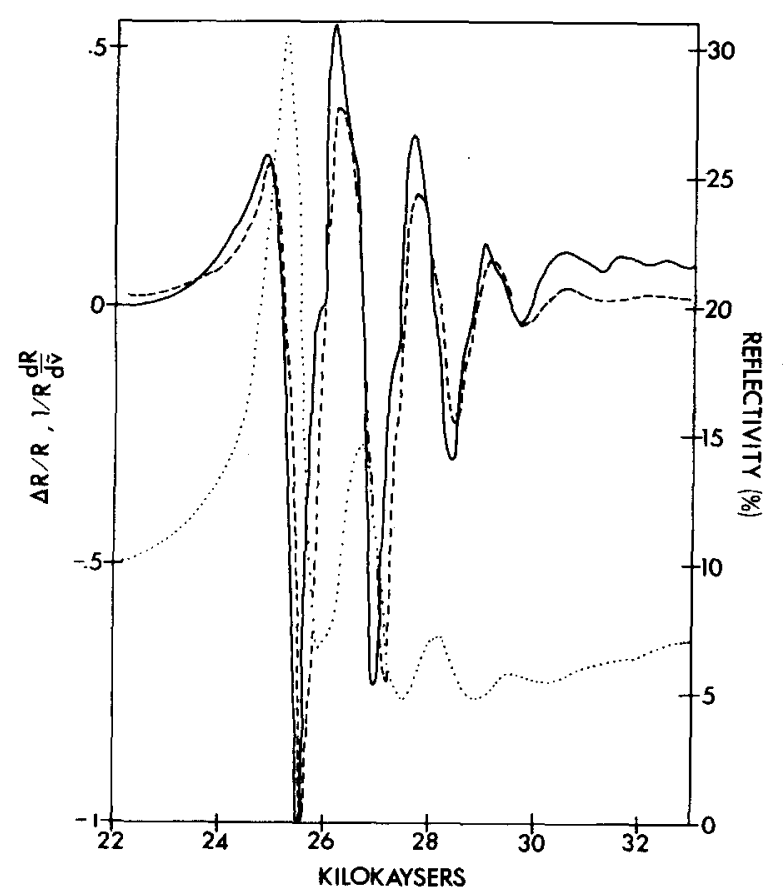

FIG. 1. Unmodulated reflection $R$ (dotted line), piezomodulated reflection spectrum $\Delta R / R$ (solid line), and calculated logarithmic derivative of the unmodulated reflection spectrum $(1 / R)(d R / d \nu)$ (dashed line) for the b polarization of (001) for anthracene at $300 \mathrm{~K} . \Delta R / R$ and $1 / R(d R / d \tilde{\nu})$ are shown normalized to facilitate comparisons.

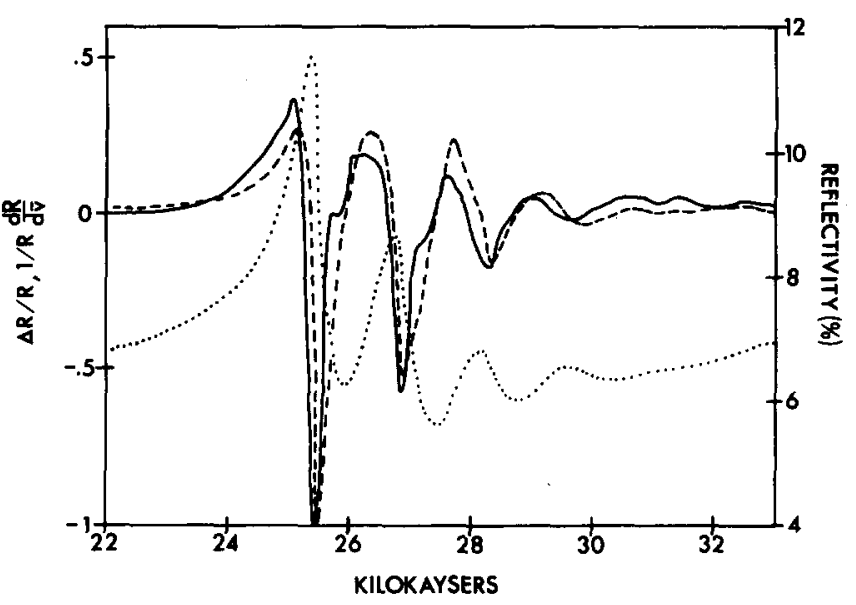

FIG. 2. $R, \Delta R / R$, and $1 / R(d R / d V)$ for the a polarization of (001) for anthracene at $300 \mathrm{~K}$. (Labeling and plot conventions as in Fig. 1.)

surface of the crystal. These features in the piezoreflection spectra of the thin anthracene platelets were further investigated by measuring the unmodulated reflection spectra of the crystal bonded to the piezoelectric transducer surface. Excluding these spectral artifacts, the measured reflection spectra are in agreement with those reported earlier. ${ }^{2}$

\section{PIEZOREFLECTION RESULTS AND DISCUSSION}

The low resolution direct reflection $R$, modulated piezoreflection $\Delta R / R$, and calculated logarithmic derivative $(1 / R)(d R / d \bar{\nu})$ spectral profiles relevant to the $b$ principal direction of the (001) face are shown in Fig. 1. The same sequence of superimposed spectral plots cor responding to the a principal direction is presented in Fig. 2. In order to facilitate comparisons between $\Delta R / R$ and $(1 / R)(d R / d \tilde{\nu})$, these spectral profiles are shown normalized in Figs. 1 and 2. The unnormalized $a$ and $b$ polarized low resolution piezoreflection spectra are shown superimposed in Fig. 3, and the corresponding high resolution $\Delta R / R$ spectra in the energy region of

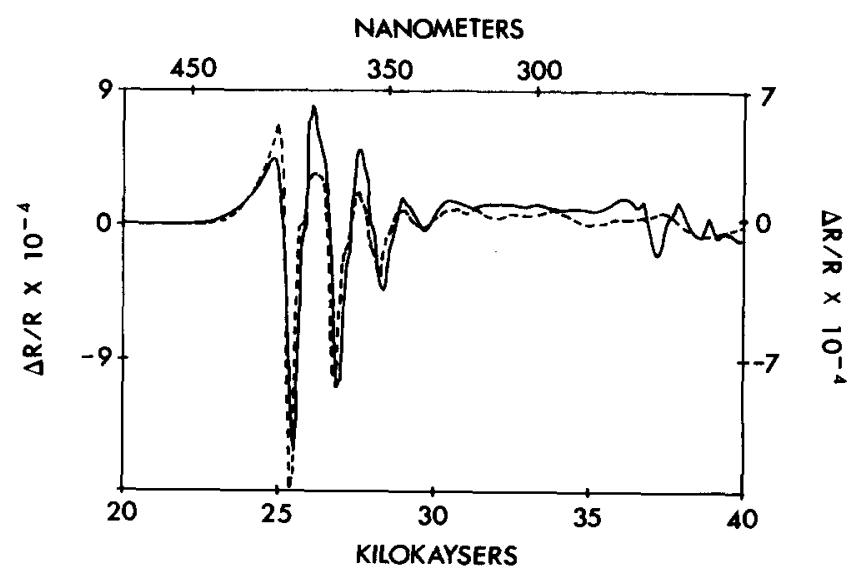

FIG. 3. b- (solid line) and a-polarized (dashed line) piezomodulated reflection spectra for (001) face of anthracene at $300 \mathrm{~K}$. 


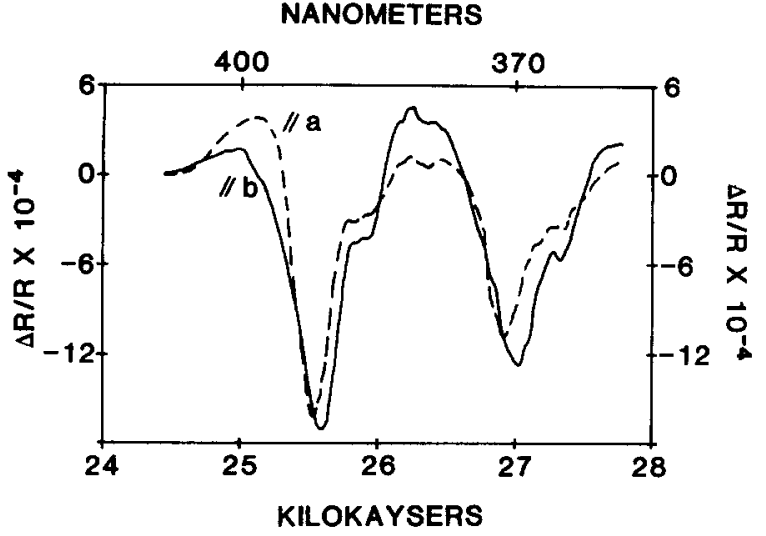

FIG. 4. High resolution $\Delta R / R$ spectra for the $(0,0)$ and $(0,1)$ vibronic bands of anthracene for the $a$ and $b$ polarizations.

the $(0,0)$ and $(0,1)$ vibronic transitions are presented in Fig. 4. The principal features present in both the low resolution piezoreflection spectra and the reflection data of Clark and Philpott ${ }^{2}$ are compiled in Table II. The principal features shown in both the high resolution piezoreflection data and the $2 \mathrm{~K}$ reflection spectra of Philpott and Turlet ${ }^{4}$ are tabulated elsewhere. ${ }^{12}$

The close correspondence between the $a$ and $b$ polarized piezoreflection spectra and the relevant logarith mic derivatives shown in Figs. 1 and 2 indicates that the principal effect of lattice strain is a shift of the vibronic exciton energies. Both the sense of the piezomodulated spectra with respect to the calculated logarithmic derivatives and the comparable relative magnitudes of the amplitudes which occur in the corresponding $\Delta R / R$ and $(1 / R)(d R / d \bar{\nu})$ profiles reveal that the vibronic exciton resonances belonging to a particular factor group component experience approximately equal strain shifts to lower energies due to crystal compression.

The b polarized $\Delta R / R$ spectrum is virtually superimposable with the corresponding logarithmic derivative in the 24000 to $32000 \mathrm{~cm}^{-1}$ energy region. The modest shifts of the amplitudes in $\Delta R / R$ relative to those in $(1 / R)(d R / d \tilde{\nu})$ are within the resolution of the derivative which was calculated numerically from the reflection spectrum of Clark and Philpott. ${ }^{2}$ Several deep minima are present in $\Delta R / R$ at $25550,26950,28380$, and $29720 \mathrm{c} \mathrm{m}^{-1}$ with corresponding magnitudes of -15.143 $\times 10^{-4},-11.104 \times 10^{-4},-4.457 \times 10^{-4}$, and $-0.444 \times 10^{-4}$, respectively. These local minima occur at energy corresponding to rapid rises in crystal reflectivity. These minima are expected to locate the $\mathbf{k}=0$ polariton (longitudinal Coulomb exciton) energies (see paper I). Prominent shoulders and inflections are evident in the $b$ polarized $\Delta R / R$ spectrum in the energy regions between successive deep minima, and the energy spacings between adjacent shoulders or inflections show that several components of the single particle vibronic exciton progression derived from the relatively intense 390 $\mathrm{cm}^{-1}$ anthracene vibration are resolved in the piezoreflection spectrum. Weak undulations superimposed on the major excursions in $\Delta R / R$ indicate the possible

TABLE II. Principal features of the anthracene reflection, piezoreflection, absorption, and piezoabsorption spectra for (001) at $300 \mathrm{~K} .^{a, b}$

\begin{tabular}{|c|c|c|c|c|c|c|c|}
\hline \multicolumn{2}{|c|}{$\|$ b axis } & \multicolumn{2}{|c|}{$\|$ a axis } & \multicolumn{2}{|c|}{$\| \mathrm{b}$ axis } & \multicolumn{2}{|c|}{ II a axis } \\
\hline $\begin{array}{c}\text { Piezoreflection } \\
\text { i }(\mathrm{kK})\end{array}$ & $\begin{array}{l}\text { Reflection }{ }^{c, d} \\
i(k K)\end{array}$ & $\begin{array}{c}\text { Piezoreflection } \\
\nu(\mathrm{kK})\end{array}$ & $\begin{array}{l}\text { Reflection }^{\mathrm{c}, \mathrm{d}} \\
\tilde{\nu}(\mathrm{kK})\end{array}$ & $\begin{array}{c}\text { Piezoabsorption } \\
\text { v } \\
\text { (kK) }\end{array}$ & $\begin{array}{c}\text { Absorption } \\
\qquad(\mathrm{kK})\end{array}$ & $\begin{array}{c}\text { Piezoabsorption } \\
\text { i (kK) }\end{array}$ & $\begin{array}{c}\text { Absorption } \\
i(\mathrm{kK})\end{array}$ \\
\hline 24.95 (max.) & & 25.05 (max.) & & 25.00 (max.) & & 25.20 (max.) & \\
\hline 25.10 (shl.) & & & & 25.10 (wk. shl.) & & & \\
\hline 25.17 (zero) & 25.22 (max.) & 25.22 (zero) & 25.37 (max.) & 25.27 (zero) & 25.31 (max.) & 25.40 (zero) & $25.20(\max )$. \\
\hline 25.55 (min.) & $(30.46 \%)$ & 25.47 (min.) & (11.44\%) & 25.40 (min.) & & 25.50 (min.) & \\
\hline 25.85 (shl.) & 25.90 (min.) & 25.83 (shl.) & 25.90 (min.) & 25.90 (min.) & 25.95 (wk. shl.) & 25.85 (shl.) & \\
\hline 26.05 (shl.) & & 26.00 (max.) & & 26.04 (zero) & 26.20 (min.) & 26.13 (zero) & 26.25 (min.) \\
\hline 26.15 (max.) & & & & 26.40 (shl.) & & 26.35 (wk. shl.) & \\
\hline 26.30 (shl.) & & & & 26.55 (max.) & & 26.60 (max.) & \\
\hline 26.67 (zero) & $26.70(\max )$. & 26.59 (zero) & $26.76(\max )$. & 26.81 (zero) & $26.82(\max )$. & 26.79 (zero) & 26.93 (max.) \\
\hline 26.95 (min.) & $(14.72 \%)$ & 26.87 (min.) & $(8.65 \%)$ & 26.90 (wk, shl.) & & & \\
\hline 27.33 (shl.) & & 27.18 (shl.) & & $27.00(\mathrm{~min})$. & & 27.00 (min.) & \\
\hline $27.65(\max )$. & 27.50 (min.) & 27.55 (max.) & 27.50 (min.) & 27.40 (shl.) & & 27.25 (shl.) & \\
\hline 27.80 (shl.) & & 27.80 (shl.) & & 27.55 (zero) & 27.70 (min.) & 27.65 (wk. infl.) & 27.80 (min.) \\
\hline 28.00 (shl.) & & & & 27.90 (max.) & & $27.90(\max )$. & \\
\hline 28.06 (zero) & $28.18(\max )$. & 27.91 (zero) & & 28.26 (zero) & $28.30(\max )$. & 28.15 (zero) & $28.36(\max )$. \\
\hline 28.38 (min.) & $(7.29 \%)$ & 28.27 (min.) & $28.20(\max )$. & 28.40 (min.) & & 28.40 (min.) & \\
\hline 28.65 (shl.) & 28.90 (min.) & 28.60 (shl.) & $(6.81 \%)$ & 28.95 (shl.) & 29.15 (min.) & 28.70 (shl.) & 29.20 (min.) \\
\hline 29.05 (max.) & & $29.00(\max )$. & 28.80 (min.) & $29.30(\max )$. & & 29.30 (max.) & \\
\hline 29.32 (shl.) & & 29.32 (shl.) & & 29.60 (zero) & $29.70(\max )$. & & 29.70 (max.) \\
\hline 29.57 (zero) & 29.55 (max.) & & & 29.90 (br. min.) & & 29.90 (br. min.) & \\
\hline 29.72 (min.) & $(5.84 \%)$ & 29.70 (min.) & $29.60(\max )$. & 31.00 (br. $\max )$. & & 30.90 (br. $\max )$. & \\
\hline 31.25 (min.) & & 31.15 (min.) & $(6.54 \%)$ & 31.35 (br. min.) & 31.10 (br. $\max )$. & $\begin{array}{l}31.45 \text { (zero) } \\
31.65 \text { (br. max.) }\end{array}$ & 31.10 (br. $\max )$. \\
\hline & & & & & & 34.50 (max.) & 34.90 (br. $\max )$. \\
\hline
\end{tabular}

a The crystal spectra were taken from Clark and Philpott (Ref. 2).

${ }^{b}$ The tabulated spectral data span the energy region corresponding to the vibronic progression of the short axis polarized, ${ }^{1} B_{2 u}-{ }^{1} A_{1}$ transition.

"Structure is designated as max. = maximum, min. = minimum, shl. = shoulder, infl. = inflection, zero =zero crossing, br. = broad, and wk. = weak.

${ }^{d}$ Percentages given in parentheses represent absolute reflectivities at $\tilde{\nu}_{\max }$. 
detection of two or multiparticle exciton states. ${ }^{13}$ These weak features and a conspicuous shoulder seen at $25100 \mathrm{~cm}^{-1}$ in the $b$ polarized spectrum but not observed in the a polarized spectrum are well defined in the high resolution data. ${ }^{12}$

It is worth calling attention to the only significant deviation between $\Delta R / R$ and $(1 / R)(d R / d \bar{\nu})$ for the $\mathrm{b}$ principal direction. As may be observed in Fig. 1, the lowest energy maximum in $\Delta R / R$ occuring at $24950 \mathrm{~cm}^{-1}$ is superimposable with the local maximum in

$(1 / R)(d R / d \bar{\nu})$. In contrast, the higher energy maxima in $\Delta R / R$ located at 26150,27650 , and $29050 \mathrm{~cm}^{-1} \mathrm{ex}-$ hibit the same trend as that shown in $(1 / R)(d R / d \bar{\nu})$ but the amplitudes of $\Delta R / R$ at the se energies are unusually large.

Although the correspondence between the a polarized low resolution $\Delta R / R$ spectrum and the relevant logarithmic derivative shown in Fig. 2 is reasonable, it is not as close as that observed for the b polarized $\Delta R / R$ and $(1 / R)(d R / d \bar{\nu})$ spectral profiles. The zero crossings of the a polarized $\Delta R / R$ spectrum in the vicinity of the $(0,0)$ and $(0,1)$ vibronic transitions are red shifted 150 and $170 \mathrm{~cm}^{-1}$, respectively, relative to the corresponding zero crossings present in $(1 / R)(d R / d \tilde{\nu})$. These shifts are greater than the uncertainty or resolution of the numerically calculated derivative. Hence, a measurable deviation exists between $\Delta R / R$ and $(1 / R)(d R / d \tilde{\nu})$ for the a principal direction. Furthermore, as indicated in Fig. 2, the maxima attained by $(1 / R)(d R / d \tilde{\nu})$ in the energy region corresponding to the $(0,0),(0,1)$, and $(0,2)$ transitions are approximately equal in magnitude. In contrast, the amplitudes of the maxima in $\Delta R / R$ occurring at 25050,26000 , and $27550 \mathrm{~cm}^{-1}$ gradually decrease with increasing energy. This deviation at the maxima in the a polarized spectrum is just the opposite of that for the $\mathrm{b}$ polarized spectrum where the $\Delta R / R$ maxima attain anomalously large values compared to $(1 / R)(d R / d \tilde{\nu})$.

The four prominent minima observed in the a polar ized $\Delta R / R$ spectrum at $25470,26870,28270$, and $29700 \mathrm{~cm}^{-1}$ have corresponding magnitudes of -13.857 $\times 10^{-4},-7.905 \times 10^{-4},-2.407 \times 10^{-4}$, and $-0.209 \times 10^{-4}$, respectively. The differences in the energies of the corresponding deep minima registered in the $a$ and $b$ polarized spectra are (for increasingly higher energy minima) $80,80,110$, and $20 \mathrm{~cm}^{-1}$, respectively. These differ little from the reported bandpass of $60 \mathrm{~cm}^{-1}$ for the low resolution experiments. This close agreement of the corresponding minima and their positions at the high energy edges of the reflection stopbands support the use of the minima to locate the $\mathbf{k}=0$ vibronic polariton energies (see paper I). The latter, in the absence of appreciable damping or spatial dispersion, are independent of either the photon polarization or the direction of $\mathbf{k}$ (for $|\mathbf{k}| \cong 0$ ).

The magnitudes of $\Delta R / R$ for the a principal direction are somewhat larger than would be anticipated from a consideration of the calculated logarithmic derivative. The amplitudes of $\Delta R / R$ are comparable to those observed for the b principal direction. Both the aforementioned deviations between $\Delta R / R$ and $(1 / R)(d R / d \bar{\nu})$ for the a principal direction and the larger than expected $\Delta R / R$ magnitudes suggest that two or more exciton modulation mechanisms are concurrently operative for the ac polarized vibronic excitons. Similar to the b polarized spectrum, prominent shoulders and reflections observed in the a polarized spectrum in the energy regions between successive deep minima may be correlated with the single particle vibronic exciton resonances based on the $390 \mathrm{~cm}^{-1}$ vibration.

A comparison between the low resolution $\mathbf{a}$ and $\mathbf{b}$ polarized piezoreflection spectra (Fig. 3) shows that the major derivative-like excursions in the a polarized spectrum are slightly shifted to the red of those observed for the b principal direction except in the vicinity of the $(0,0)$ vibronic transition. This is attributable to the deviations in the a polarized spectrum from a strict transition energy derivative. Although the energy separations in either spectrum between successive maxima, minima, inflections, and shoulders afford the recognition of the $\approx 1400$ and $390 \mathrm{~cm}^{-1}$ single particle vibronic exciton progressions, absolute energy assignments are made less certain by the slight deviations of the spectra from strict transition energy derivatives. In this regard, if the zero crossings locate the vibronic exciton energies of the $\approx 1400 \mathrm{~cm}^{-1}$ progression, then the cal culated factor group splittings of all but that of the $(0,0)$ transitions would be opposite in sign to those deduced from the Kramers-Kronig transformed absorption spectra. Hence, the deviations in $\Delta R / R$ from $(1 / R)(d R / d \bar{\nu})$ render the task of determining the Davydov splittings difficult from the directly measured data. However, it must be recognized that it is generally unwise to base absolute energy assignments solely on either $R$ or $\Delta R / R$ data since analysis can be made with certainty only from the fundamental optical constants.

Calculation of the crystal oscillator strengths and polarization ratios for the first singlet system have been made reasonably successful by including mixing with the $\hat{L}_{A}$ polarized second singlet. Thus, the observed deviations in the a polarized $\Delta R / R$ spectrum from a strict derivative may be attributable to a strain modulation of the coupling between the ac polarized factor group component and the $\mathrm{L}_{\mathrm{A}}$ polarized transition; the strain modulates both the exciton energies and the oscillator strengths and hence the band shapes.

Another source of the observed deviations may be found in the presence of the heretofore undetected ( $\mathrm{cf}$. $\mathrm{Clar}^{14}$ and Bree et al. ${ }^{15}$ ) long axis polarized, ${ }^{1} L_{b}-{ }^{1} A_{18}$ transition which is believed to occur in the same energy region as the $S_{1} \hat{\mathbf{M}}_{\mathrm{A}}$ polarized transition. The projected intensity of the ${ }^{1} L_{b}$ transition would result in an increase in the measured integrated intensity of the a polarized spectrum and hence a decrease in the $b / a$ polarization ratio. Furthermore, the measured $\Delta R / R$ spectrum in an energy region comprised of two superimposed band systems would not, in general, exhibit the local maxima, minima, and zero crossings which would result from the strain modulation of a single, rigidly shifted band system. This obtains for the general case of two superimposed band systems which are characterized by different logarithmic derivatives and strain-induced 
energy shifts. Although no other definitive evidence is presently available to support the existence of the ${ }^{1} L_{b}$ transition in pure crystalline anthracene, the presence of this band system would explain the observed $\Delta R / R$ spectrum for the a polarized spectrum.

The high resolution piezoreflection spectra for the a and $b$ principal directions of the (001) face at $290 \mathrm{~K}$ in the energy region corresponding to the $(0,0)$ and $(0,1)$ vibronic transitions are shown superimposed in Fig. 4. The calculated energy spacings between successive inflections and shoulders exhibited by the $\Delta R / R$ spectra reveal the single particle vibronic exciton progressions based on the totally symmetric 1400 and $390 \mathrm{~cm}^{-1}$ vibrations of anthracene, ${ }^{15}$ where a considerable amount of structure in the form of weak shoulders, inflections, and sharp peaks is superimposed on the major excursions in $\Delta R / R$ due to the 1400 and $390 \mathrm{~cm}^{-1}$ single particle progressions. The elucidation of structure which may be correlated with the $390 \mathrm{~cm}^{-1}$ progression is comparable to that reported from the 2 and $10 \mathrm{~K}$ reflection spectra respectively..$^{3,4}$ The effective enhancement of spectral features apparent in the $\Delta R / R$ spectra has not been heretofore realized in any other type of ambient temper ature spectrum. This definition of structure is partially attributable to the high detection sensitivity of the strain-induced fractional reflectivity changes $\left(\approx 5 \times 10^{-6}\right)$, which facilitates the detection of weak structure which is normally obscured in an unmodulated spectrum by the presence of unavoidable fluctuations in the optical and electronic instrumentation. The enhancement of structure is also aided by the existence of differences in the modulation sensitivities of the exciton resonances. The relative strain shifts of different single or multiparticle manifolds of states facilitate the recognition of weakly perturbed resonances which normally appear in the unmodulated spectrum as shoulders or slight inflections superimposed on an intense background. Furthermore, if the strain modulation of exciton-phonon interactions which dampen (and hence broaden) the crystal excitations is small, then a measurement of the differential changes in crystal reflectivity due to an extensive-compressive strain cycle will result in the decrease of a background signal arising from the scatttering of excitons by lattice phonons.

Prior to commenting on the nature of the weak spectral features registered in $\Delta R / R$, it is useful to extract some quantitative information from the major excur sions of $\Delta R / R$ in the energy region corresponding to the ac and $b$ polarized factor group components of the $(0,0)$ transition. The deep minima at 25590 and $25530 \mathrm{~cm}^{-1}$ observed in the $b$ and a polarized spectra, respectively, are split by only $60 \mathrm{~cm}^{-1}$. This is in reasonably good agreement with the value of $80 \mathrm{~cm}^{-1}$ given by Morris and Sceats $^{3}$ from the room temperature spectral data. These workers also report that the splitting decreases to $10 \mathrm{~cm}^{-1}$ at $10 \mathrm{~K}$. The decrease in the $\mathrm{k}=0$ splitting due to crystal cooling may be a manifestation of weaker polariton-phonon coupling at lower temperatures. From the zero crossings in $\Delta R / R$ at 25130 and $25310 \mathrm{~cm}^{-1}$ for the $b$ and a principal directions, respectively, and the aforementioned $\mathbf{k}=0$ polariton energies, one deduces a factor group splitting of $180 \mathrm{~cm}^{-1}$ and polariton stop bandwidths of 460 and $220 \mathrm{~cm}^{-1}$ for the $b$ and a polarized factor group components, respectively. The foregoing implicitly assumes that the zero crossings in $\Delta R / R$ are a reasonably accurate indication of the $\mathrm{k} \rightarrow \infty$ polariton energies. The above values are in good agreement with the Davydov splitting for the $(0,0)$ transition of $210 \mathrm{~cm}^{-1}$ and the stop bandwidths of $465 \mathrm{~cm}^{-1}$ (for b) and $330 \mathrm{~cm}^{-1}$ (for a) from the $198 \mathrm{~K}$ spectra. ${ }^{3}$ A straightforward analysis similar to the foregoing for the $(0,1)$ component of the $1400 \mathrm{~cm}^{-1}$ progression is precluded by the great deal of spectral congestion in this energy region and the more pronounced deviations in the a polarized spectrum from a strict transition energy derivative.

Remarkable correspondence of the principal features in the high resolution $\Delta R / R$ spectrum and the $2 \mathrm{~K}$ reflec tion spectrum has been previously reported. ${ }^{12}$ The prominent reflection maxima at $2 \mathrm{~K}$ corresponding to the $b$ polarized $(0,0)$ and $(0,1)$ vibronic transitions (for the $1400 \mathrm{~cm}^{-1}$ progression) are red shifted 113 and 110 $\mathrm{cm}^{-1}$, respectively, relative to the maxima in the $298 \mathrm{~K}$ spectrum. ${ }^{2}$ Similarly, the a polarized reflection maxima at $2 \mathrm{~K}$ corresponding to the $(0,0)$ and $(0,1)$ transi tions are red shifted 51 and $65 \mathrm{~cm}^{-1}$, respectively, relative to that observed at ambient temperature. These red shifts, which are primarily attributable to a compression of the lattice upon cooling, are somewhat smaller than those obtained from the $\epsilon_{i}$ spectral data. ${ }^{3}$ Thus, the comparisons between the $\Delta R / R$ and $2 \mathrm{~K}$ spectra are only approximate. However, there is remarkable agreement between features observed in the $298 \mathrm{~K}$ $\Delta R / R$ spectra and those found in the $2 \mathrm{~K}$ reflection spectra. This may be an indication that structure oc curs in the piezoreflection spectra at energies corresponding to the bare exciton resonances.

A rather abrupt change in the curvature of the $\Delta R / R$ spectral profile for the $b$ polarized spectrum occurs at $25450 \mathrm{~cm}^{-1}$ or approximately $320 \mathrm{~cm}^{-1}$ to the blue of the $(0,0)$ transition origin, which is taken to be the zero crossing in $\Delta R / R$ at $25130 \mathrm{~cm}^{-1}$. Both the magnitude of the energy spacing and the correlation which may be established with structure appearing at approximately the same energy in the $2 \mathrm{~K}$ reflection spectrum strongly indicate that this inflection locates the lower edge of the two particle exciton band corresponding to a $(0,0)$ vibronic exciton and a $390 \mathrm{~cm}^{-1}$ vibrational exciton. A two or multiparticle excitation is comprised of a resonating vibronic exciton and one or more vibrational excitons which are assumed to be nonpropagating. ${ }^{12}$ The creation of a two particle state is a second order process wherein a single particle excitation decays into a two particle exciton. Consequently, such resonances are only weakly evident in the exciton spectrum except at energies corresponding to peaks, troughs, or inflection points in the joint density of exciton states, i.e., critical points. Hence, abrupt changes in slope or sharp singular struc ture in $\Delta R / R$ can locate thresholds in the two or multiparticle exciton joint density of states. ${ }^{16}$ Common to the a and b polarized $\Delta R / R$ spectra are weak, albeit sharp, features in the $\approx 25800$ to $25980 \mathrm{~cm}^{-1}$ and in the $\approx 26100$ to $26700 \mathrm{~cm}^{-1}$ energy regions. This structure correlates with that appearing in the $2 \mathrm{~K}$ reflection spectra and is tentatively assigned to two and multi- 


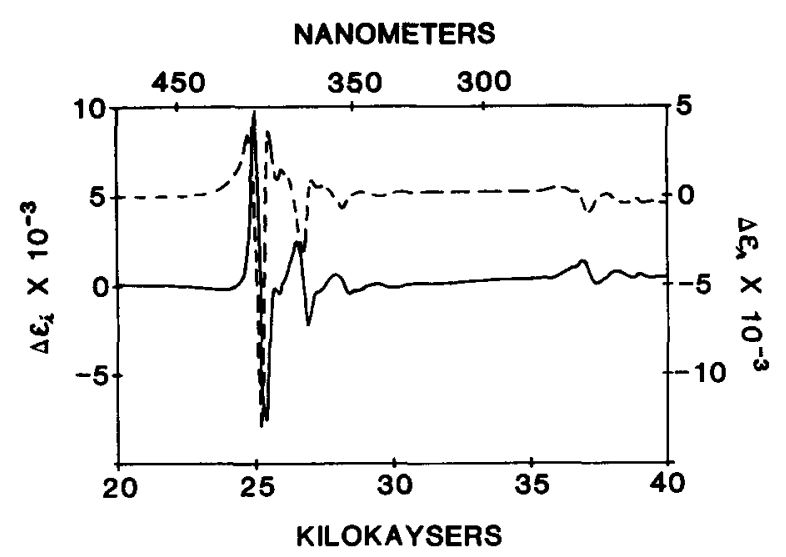

FIG. 5. $\Delta \epsilon_{r}$ (differential real part of dielectric function; dashed line) and $\Delta \epsilon_{i}$ (differential imaginary part of dielectric function; solid line) for the b polarization of (001) of anthracene.

particle exciton resonances.

As discussed in paper I, the Seraphin coefficients are ill-behaved functions for anthracene. Hence, analy sis of the data using these functions is not useful.

\section{DIFFERENTIAL KRAMERS-KRONIG TRANSFORMS-PIEZOSPECTROSCOPIC DATA}

The piezomodulation spectra derived from both of the Kramers-Kronig transforms of the low resolution $\Delta R / R$ data are introduced with the superimposed spec tral profiles of $\Delta \epsilon_{r}$ and $\Delta \epsilon_{i}$ and $\Delta a$ and a corresponding to the $b$ principal direction of the (001) face in Figs. 5 and 6 , respectively. The same sequence of spectral plots relevant to the a principal direction of the (001) face is presented in Figs. 7 and 8 . The superimposed $a$ and $b$ polarized $\Delta \epsilon_{i}$ spectra are shown in Fig. 9, whereas a similar superposition of the $\Delta \epsilon_{r}$ spectral profiles is given in Fig. 10.

The integral transforms of the low resolution $\Delta R / R$ data wherefrom the $\Delta \theta$ spectral profiles are calculated were obtained utilizing discrete data sets with a resolution of $100 \mathrm{~cm}^{-1}$. The latter, which involves some loss in resolution in the transformed spectra relative to that associated with the $\Delta R / R$ spectra, was limited by

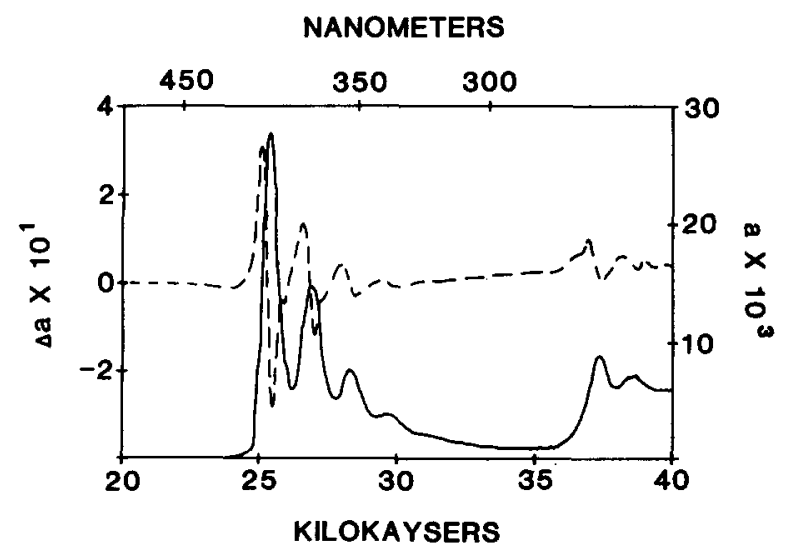

FIG. 6. $a$ (solid line) and $\Delta a$ (dashed line) spectra for the b polarization of (001) of anthracene.

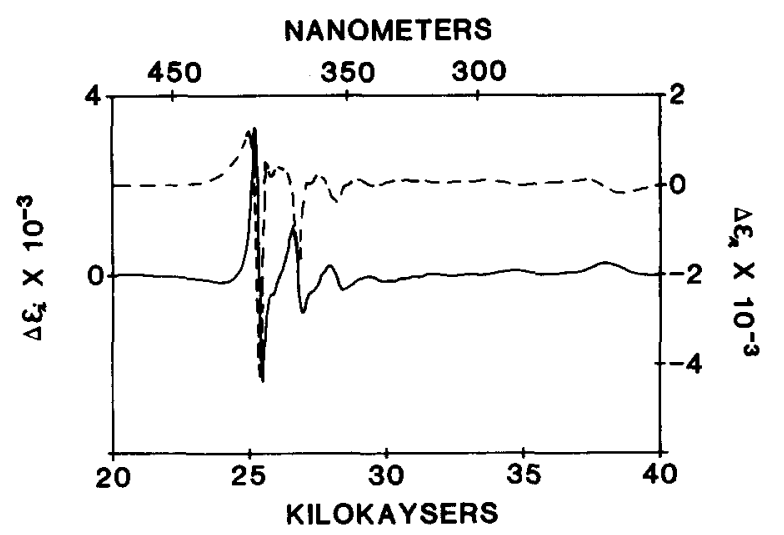

FIG. 7. $\Delta \epsilon_{r}$ and $\Delta \epsilon_{i}$ for the a polarization of (001) of anthracene (conventions as in Fig. 5).

the resolution of the available $n$ and $\kappa$ data. ${ }^{2}$ The Kramers-Kronig transforms of the high resolution piezoreflection spectra were precluded by both the unavailability of high resolution, low temperature $n$ and $\kappa$ data and the rather limited energy range spanned by the $\Delta R / R$ data.

The presence of exceptionally small background signals in the anthracene piezoreflection spectra affords an opportunity to test the efficacy of the differential Kramers-Kronig transform methods. Transform-induced spectral artifacts and offset signals can be easily recognized. Criteria for acceptability of the calculation of differential transforms were empirically established by performing the $\Delta R / R$ transforms in a variety of ways. Consistent with this objective, the averaged low resolution piezoreflection spectra for both the $\mathbf{a}$ and $\mathbf{b}$ principal directions were transformed using three different procedures. One type of transform was calculated from a $\Delta R / R$ spectrum which was smoothly extrapolated to the zero signal level in the region to the blue of the highest energy experimental data point at $40000 \mathrm{~cm}^{-1}$. The second type of integral transform entailed addition of various derivative-like excursions to the measured $\Delta R / R$ spectrum in the 40000 to $50000 \mathrm{~cm}^{-1}$ energy region. These represented an attempt to simulate the piezore flection response function at photon energies in the re gion of the long axis polarized, ${ }^{1} B_{1 u}-{ }^{1} A_{1}$ transition.

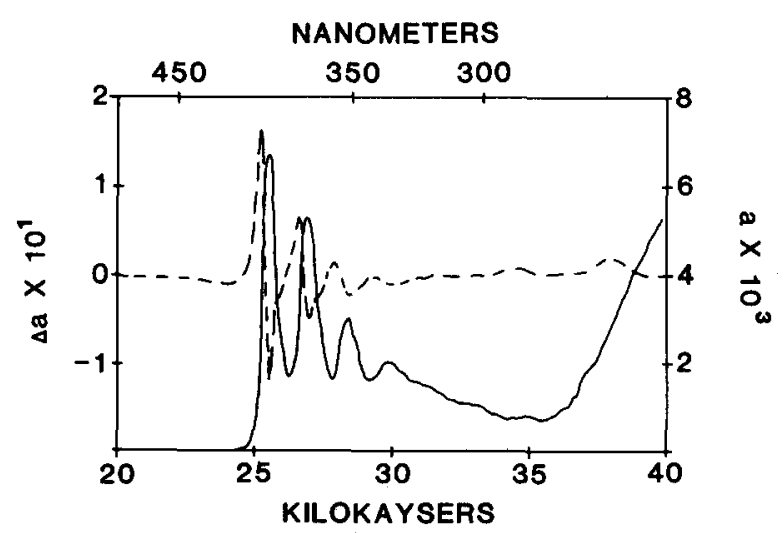

FIG. 8. $a$ and $\Delta a$ spectra for the a polarization of (001) of anthracene (conventions as in Fig. 6). 


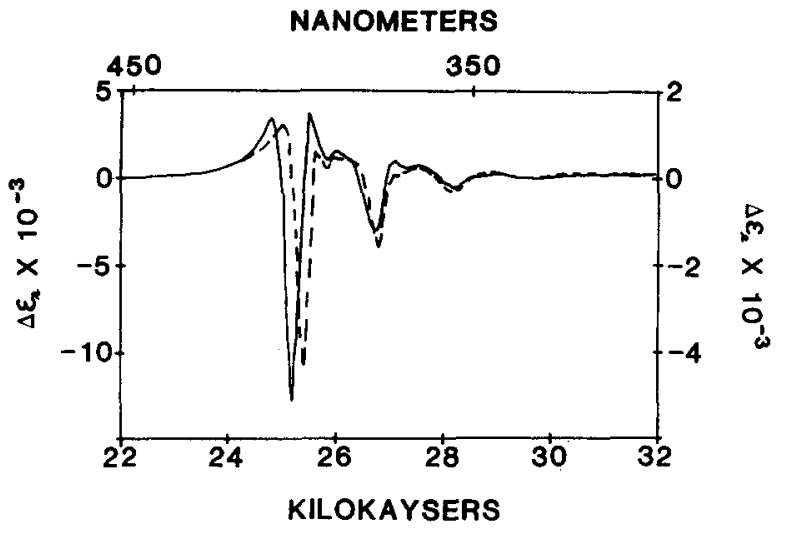

FIG. 9. $\Delta \epsilon_{\text {, for }} \mathrm{b}$ polarization (solid line) and a polarization (dashed line).

The third type of Kramers-Kronig transform involved integrating only the experimentally measured $\Delta R / R$ spectrum spanning the 22000 to $40000 \mathrm{~cm}^{-1}$ energy range, i.e., the region between which $\Delta R / R$ asymptotically approaches zero on the red and blue sides. A single important conclusion can be drawn from the results of the three methods of calculating the transformations. The integral transforms of the modified or embellished $\Delta R / R$ spectra results in $\Delta \epsilon_{\varphi}$ and $\Delta \epsilon_{i}$ spectra negligibly different from those calculated using only the measured $\Delta R / R$ data sets. This empirical approach indicates that the differential transforms are insensitive to the assumed (or neglected) variations of $\Delta R / R$ in energy regions not experimentally accessible and which are beyond regions in which the $\Delta R / R$ has asymptotically fallen to zero. Thus, the piezomodulation spectra derived from transforms utilizing only the measured data were taken to be optimal. These relatively straightforward differential Kramers-Kronig transform procedures contrast with those associated with the transformation of unmodulated reflection spectra wherein the assumed dependence of $R$ in energy regions not experimentally accessible is occasionally quite crucial. The quality of a differential transform may be partially judged by considering the spectral variation registered in $\Delta \theta$. As may be observed in Figs. 11 and 12 , the features in the $\Delta \theta$ spectra profiles are clearly

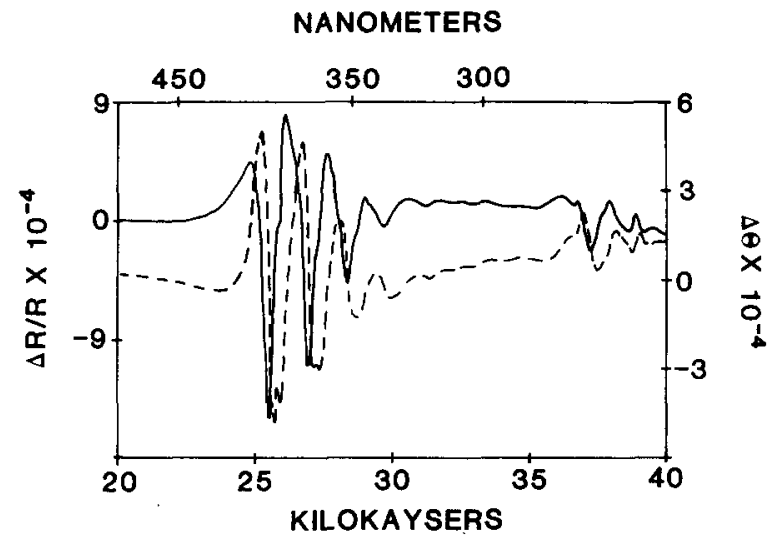

FIG. 11. $\Delta R / R$ (solid line) and differential phase shift $\Delta \theta$ (dashed line) for $b$ polarization of $(001)$ of anthracene.

recognizable integral inversions of corresponding structure present in the piezoreflection spectra. Further more, the magnitude of $\Delta \theta$ decreases smoothly to zero in the energy region to the red of the absorption threshold.

As may be deduced from the listings of the principal features observed in the $a$ and $b$ polarized piezoreflection and piezoabsorption spectra (cf. Table II), the differential transform procedures result in only modest energy shifts of the structure in the $\Delta R / R$ spectra. Consequently, several comments concerning $\Delta \epsilon_{i}$ suffice to complete the discussion of the piezomodulation data.

The $\mathrm{b}$ polarized $\Delta \epsilon_{i}$ spectrum corresponds very closely to a transition energy derivative of the unmodulated crystal absorption spectrum. In contrast, the a polarized $\Delta \epsilon_{i}$ spectral profile exhibits inflections or zero crossings which are perceptibly red shifted relative to the corresponding maxima observed in $\epsilon_{i}$. The latter arises from the deviations in $\Delta R / R$ from $(1 / R)(d R / d \bar{\nu})$ for the a principal direction. However, the factor group splittings of approximately 165 and $50 \mathrm{~cm}^{-1}$ for the $(0,0)$ and $(0,1)$ components of the $1400 \mathrm{~cm}^{-1}$ progression determined from $\Delta \epsilon_{i}$ compare with those re ported by Clark and Philpott ${ }^{2}$ of 190 and $110 \mathrm{~cm}^{-1}$, respectively.

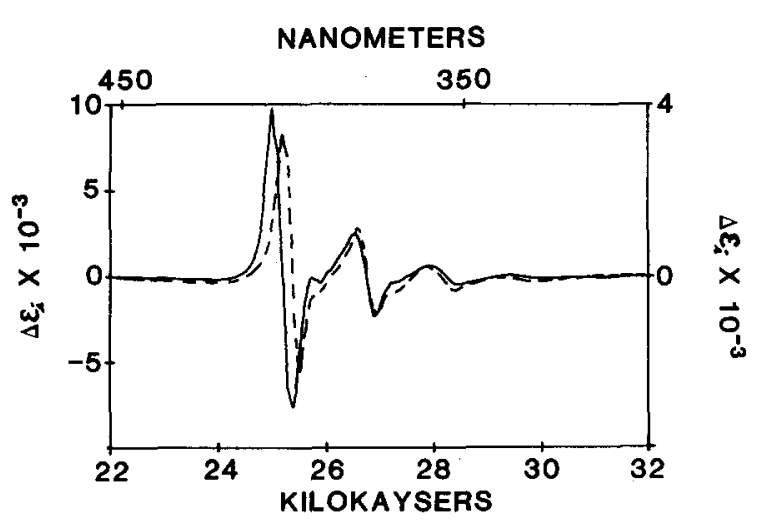

FIG. 10. $\Delta \epsilon_{i}$ for the $b$ polarization (solid line) and a polarization (dashed line).

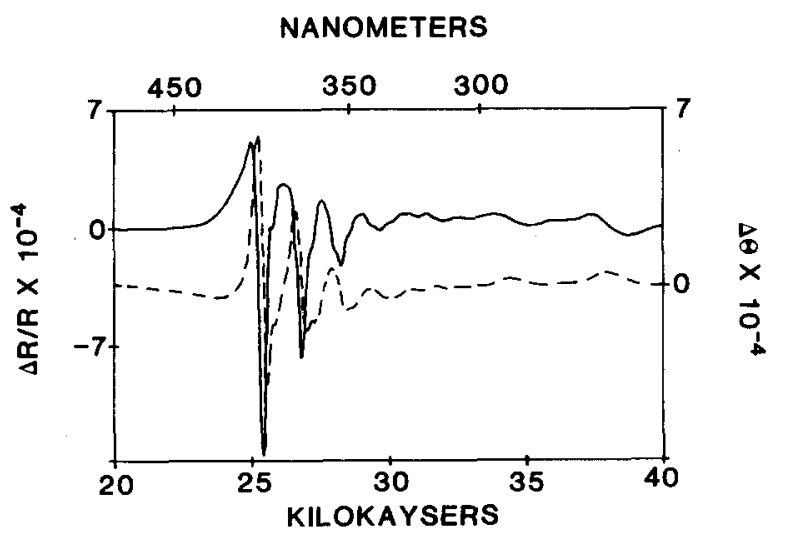

FIG. 12. $\Delta R / R$ (solid line) and $\Delta \theta$ (dashed line) for a polarization of (001) of anthracene. 
TABLE III. Strain-induced exciton energy shifts obtained from piezomodulation spectroscopya and high pressure optical spectroscopy ${ }^{b}$ for the first singlet system of anthracene.

\begin{tabular}{|c|c|c|c|c|c|c|c|c|c|c|}
\hline \multirow{2}{*}{$\begin{array}{c}\text { Vibronic } \\
\text { transition } \\
(u, r)\end{array}$} & \multicolumn{3}{|c|}{$\begin{array}{l}\text { ॥ b Polarized vibronic } \\
\text { exciton strain shifts }\end{array}$} & \multicolumn{3}{|c|}{$\begin{array}{l}\text { ac Polarized vibronic } \\
\text { exciton strain shifts }\end{array}$} & \multicolumn{3}{|c|}{$\begin{array}{l}\text { Mean exciton strain shifts } \\
\Delta E_{\text {mean }}^{u, r}=\frac{1}{2}\left(\Delta E_{*}^{u, r}+\Delta E_{+}^{u, r}\right)\end{array}$} & \multirow[b]{2}{*}{$\begin{array}{l}\Delta E_{\text {mipan }}^{u, r} \mathrm{~b} \\
\left(\mathrm{~cm}^{-1}\right)\end{array}$} \\
\hline & $\begin{array}{l}\Delta E_{\cdot}^{u, r d} \\
\left(\mathrm{~cm}^{-1}\right)\end{array}$ & $\begin{array}{l}\Delta E_{-}^{u, r e} \\
\left(\mathrm{~cm}^{-1}\right)\end{array}$ & $\begin{array}{l}\Delta E^{u, r t} \\
\left(\mathrm{~cm}^{-1}\right)\end{array}$ & $\begin{array}{l}\Delta E_{+}^{u, r d} \\
\left(\mathrm{~cm}^{-1}\right)\end{array}$ & $\begin{array}{l}\Delta E_{+}^{u, r \theta} \\
\left(\mathrm{cm}^{-1}\right)\end{array}$ & $\begin{array}{l}\Delta E_{+}^{u, r f} \\
\left(\mathrm{~cm}^{-1}\right)\end{array}$ & $\begin{array}{l}\Delta E_{\text {mean }}^{u, r} \mathrm{~d} \\
\left(\mathrm{~cm}^{-1}\right)\end{array}$ & $\begin{array}{l}\Delta E_{\text {moan }}^{u, r} \oplus \\
\left(\mathrm{cm}^{-1}\right)\end{array}$ & $\begin{array}{l}\Delta E_{\text {mean }}^{u, r} t \\
\left(\mathrm{~cm}^{-1}\right)\end{array}$ & \\
\hline$(0,0)$ & 0.54 & 0.59 & 0.65 & 1.09 & 0.88 & 1.09 & 0.82 & 0.74 & 0.87 & 0.88 \\
\hline$(0,1)$ & 0.63 & 0.59 & 0.73 & 0.84 & 0.82 & 0.83 & 0.74 & 0.71 & 0.78 & 0.78 \\
\hline$(0,2)$ & 0.74 & 0.63 & 0.62 & 0.76 & 0.70 & 0.71 & 0.75 & 0.67 & 0.67 & 0.69 \\
\hline$(0,3)$ & 0.57 & 0.62 & 0.56 & 0.64 & 0.66 & 0.58 & 0.61 & 0.64 & 0.57 & 0.68 \\
\hline Average & 0.62 & 0.61 & 0.64 & 0.83 & 0.77 & 0.80 & & & & \\
\hline
\end{tabular}

${ }^{a}$ The tabulated exciton energy shifts obtained from piezomodulation spectroscopy correspond to a planar [(001) face $]$, isotropic extensive crystal strain of $6.33 \times 10^{-5}$ or lattice parameter changes of $\Delta a=5.42 \times 10^{-4} \AA$ and $\Delta b=3.82$ $\times 10^{-4} \AA$. An average equivalent pressure required to produce the above changes in $a$ and $b$ may be calculated from the pressure dependence of the anthracene lattice parameters given by Elnahwy et al. (Ref. 10) viz., $\partial a / \partial P$ $=-0.042 \AA / \mathrm{kbar}$ and $\partial b / \partial P_{=}-0.0172 \AA \mathrm{A} / \mathrm{kbar}$. The equivalent pressure is calculated to be $17.33 \mathrm{~atm}$.

${ }^{b}$ These mean exciton strain shifts $\Delta E_{\operatorname{mean}}^{u, r}$ were calculated from the high pressure absorption spectroscopy data obtained for crystalline anthracene by Wiederhorn and Drickamer (Ref. 17). These workers report mean vibronic exciton pressure shifts of $\Delta E_{\text {mam }}^{u, r}=-K^{u, r} P$, where $K^{u, r}=0.051,0.045,0.040$, and $0.039 \mathrm{~cm}^{-1} /$ atm for the $(0,0),(0,1)$, $(0,2)$, and $(0,3)$ transitions, respectively. The tabulated mean shifts were obtained from these values of $K^{u, r}$ and the equivalent pressure of $17.33 \mathrm{~atm}$ given in the above footnote.

The estimated error in the strain shift is $\pm 0.1 \mathrm{~cm}^{-1}$.

These shifts were calculated by averaging the two values of the $\Delta R / R$ to $(1 / R)(d R / d \tilde{D})$ ratio evaluated at the energies corresponding to the peaks and troughs of the two spectral profiles in the vicinity of the $u, r$ transition.

-These shifts were calculated from the ratio of the change in $\Delta R / R$ measured from peak to trough in the vicinity of the $u, r$ transition to the corresponding change in $(1 / R)(d R / d \tilde{v})$.

'These shifts were calculated from the piezoabsorptivity data $\Delta a$ and the numerical derivatives of the crystal absorptivity $d a / d \nu$ in a manner analogous to that described in footnote $c$.

\section{MEASURED AND CALCULATED EXCITON STRAIN SENSITIVITIES}

The strain-induced exciton energy shifts determined from the piezospectroscopic investigation of the first singlet system of crystalline anthracene are compiled in Table III. The latter may be readily compared with the strain shifts in Table IV which were calculated using the dispersion and exciton resonance energy derivatives reported by Schipper. ${ }^{5}$ The measured and calculated exciton-strain shifts presented in Tables III and IV correspond to a planar [(001) face], isotropic, extensive crystal strain of $6.33 \times 10^{-5}$. Hence, the numbers compiled in the tables are positive (blue shifts) for crystal extension or, equivalently, negative (red shifts) for lattice compression. Unless otherwise noted, the discussion of exciton strain shifts will implicitly assume extensive crystal strains, or equivalently, only the absolute values of the strain shifts will be given. The calculative procedures used to deduce the strain shifts from the experimental data have been detailed in the first paper of this series.

It is important to comment on the vibronic excitonstrain shifts obtained from piezomodulation spectroscopy prior to establishing comparisons with other reported values. The $b$ and ac polarized and mean strain shifts were calculated from the piezoreflection spectra using two different procedures. The first method consisted of shifts calculated by averaging the two values of the $\Delta R / R$ to $(1 / R)(d R / d \tilde{\nu})$ ratio evaluated at the energies corresponding to the peaks and troughs of the two spectral profiles in the vicinity of the $0,0-u, r$ transition. The second method consisted of shifts calculated from the ratio of the change in $\Delta R / R$ measured from peak to trough in the vicinity of the $0,0 \rightarrow u, r$ transition to the corresponding change in $(1 / R)(d R / d \tilde{\nu})$. Strain shifts from the piezoabsorption data were obtained by calculation from the piezoabsorptivity data $\Delta a$ and the numerical derivatives of the crystal absorptivity $d a / d \bar{\nu}$ in a manner analogous to that described in the first method. The triples of numbers constituting the different values calculated for the strain shift of a particular vibronic exciton resonance exhibit variances which are comparable to the estimated error in the shift of $\sim \pm 0.1 \mathrm{~cm}^{-1}$. These relatively small variances show that the values for the strain-induced resonance shifts are only weakly dependent on the nature of the data from which they were derived. This may be taken as an indication of the validity of the differential transform procedures.

The values corresponding to the $b$ polarized vibronic exciton-strain shifts do not reveal the clear trend in the modulation sensitivities which would be anticipated from the weak coupling exciton energy formulas. This is primarily a consequence of the deviations of $\Delta R / R$ from $(1 / R)(d R / d \nu)$, which are most pronounced at the maxima in $\Delta R / R$ in the vicinity of the $(0,1)$ and $(0,2)$ transitions. In contrast, the values for the ac polarized vibronic exciton-strain shifts indicate that the modulation sensitivities decrease for higher components of the progression. Caution should be exercised in light of the easily recognizable deviations between $\Delta R / R$ and $(1 / R)(d R / d \nu)$ for the a principal direction. The small but significant deviations which exist between both the a and $b$ polarized piezomodulation spectra and the corresponding energy derivatives present strict applicability of the model used in the derivation of the calculative 
TABLE IV. Calculated exciton-strain shifts for the first singlet system of anthracene. ${ }^{2}$

\begin{tabular}{|c|c|c|c|c|c|c|c|}
\hline \multirow{2}{*}{$\begin{array}{c}\text { Vibronic } \\
\text { transition } \\
(u, r) \\
\end{array}$} & \multicolumn{7}{|c|}{ Strain shifts for the - or $\| b$ polarized vibronic exciton states ${ }^{b}$} \\
\hline & $\begin{array}{l}\Delta E_{-r}^{u, r} \\
\left(\mathrm{~cm}^{-1}\right)\end{array}$ & $\begin{array}{l}\Delta E_{-r}^{u, r}(a) \\
\left(\mathrm{cm}^{-1}\right)\end{array}$ & $\begin{array}{l}\Delta E_{-}^{u_{r} r}(b) \\
\left(\mathrm{cm}^{-1}\right)\end{array}$ & $\begin{array}{l}\Delta D_{-}^{u, r}(a)^{\mathrm{c}} \\
\left(\mathrm{cm}^{-1}\right)\end{array}$ & $\begin{array}{l}\Delta D_{-}^{\mu, r}(b)^{\mathrm{c}} \\
\left(\mathrm{cm}^{-1}\right)\end{array}$ & $\begin{array}{l}\Delta \mathrm{I}_{-}^{\boldsymbol{u}, r}(\mathbf{k}, a)^{\mathrm{d}} \\
\left(\mathrm{cm}^{-1}\right)\end{array}$ & $\begin{array}{l}\Delta \mathrm{I}_{-}^{u, r}(\mathbf{k}, b)^{\mathrm{d}} \\
\left(\mathrm{cm}^{-1}\right)\end{array}$ \\
\hline$(0,0)$ & 0.784 & 0.437 & 0.347 & 0.396 & 0.319 & 0.041 & 0.028 \\
\hline$(0,1)$ & 0.784 & 0.437 & 0.347 & 0.396 & 0.319 & 0.041 & 0.028 \\
\hline$(0,2)$ & 0.763 & 0.425 & 0.338 & 0.396 & 0.319 & 0.029 & 0.019 \\
\hline$(0,3)$ & 0.736 & 0.409 & 0.328 & 0.396 & 0.319 & 0.012 & 0.008 \\
\hline$(0,4)$ & 0.727 & 0.403 & 0.323 & 0.396 & 0.319 & 0.007 & 0.004 \\
\hline \multirow[t]{3}{*}{ Average } & 0.759 & 0.422 & 0.337 & 0.396 & 0.319 & 0.026 & 0.017 \\
\hline & \multicolumn{7}{|c|}{ Strain shifts for the + or ac polarized vibronic exciton states ${ }^{b}$} \\
\hline & $\begin{array}{c}\Delta E_{+}^{u, r} \\
\left(\mathrm{~cm}^{-1}\right)\end{array}$ & $\begin{array}{l}\Delta E_{+}^{u, r}(a) \\
\left(\mathrm{cm}^{-1}\right)\end{array}$ & $\begin{array}{l}\Delta E_{+}^{u, r}(b) \\
\left(\mathrm{cm}^{-1}\right)\end{array}$ & $\begin{array}{l}\Delta D_{+}^{\mu, r}(a)^{\mathrm{c}} \\
\left(\mathrm{cm}^{-1}\right)\end{array}$ & $\begin{array}{l}\Delta D_{+}^{\mu, r}(b)^{\mathrm{c}} \\
\left(\mathrm{cm}^{-1}\right)\end{array}$ & $\begin{array}{l}\Delta \mathbf{I}_{+}^{u, r}(\mathbf{k}, a)^{\mathrm{d}} \\
\left(\mathrm{cm}^{-1}\right)\end{array}$ & $\begin{array}{l}\Delta I_{\stackrel{+}{u}, r}^{u}(k, b)^{d} \\
\left(\mathrm{~cm}^{-1}\right)\end{array}$ \\
\hline$(0,0)$ & 0.767 & 0.375 & 0.392 & 0.396 & 0.319 & -0.022 & 0.073 \\
\hline$(0,1)$ & 0.767 & 0.375 & 0.392 & 0.396 & 0.319 & -0.021 & 0.073 \\
\hline$(0,2)$ & 0.751 & 0.382 & 0.369 & 0.396 & 0.319 & -0.014 & 0.050 \\
\hline$(0,3)$ & 0.731 & 0.390 & 0.341 & 0.396 & 0.319 & -0.006 & 0.022 \\
\hline$(0,4)$ & 0.724 & 0.393 & 0.331 & 0.396 & 0.319 & -0.003 & 0.012 \\
\hline Average & 0.748 & 0.383 & 0.365 & 0.396 & 0.319 & -0.013 & 0.046 \\
\hline
\end{tabular}

${ }^{a}$ The shifts were calculated using the dispersion and exciton resonance energy derivatives reported in Table 7 of the theory paper presented by Schipper. ${ }^{5}$ A description of the calculations and all of the relevant equations may be found in paper $I$. The tabulated energy shifts correspond to a planar [(001) face], isotropic, extensive crystal strain of $6.33 \times 10^{-5}$ or lattice parameter changes of $\Delta a$ $=5.42 \times 10^{-4} \AA$ and $\Delta b=3.82 \times 10^{-4} \AA$. The contributions to the exciton shifts due to other crystal strains were not evaluated.

bThe $(a)$ and $(b)$ designations for $\Delta E, \Delta D$, and $\Delta I$ correspond to $\|$ a-axis and $\|$ b-axis strains, respectively.

The calculated $\Delta D$ values are based on an assumed site shift of $-1.98 \mathrm{kK}$. This value was used by Schipper (1974) to parametrize the dispersion derivative lattice sums.

The $\Delta \mathrm{I}$ values include contributions due to the mixing of the first and second singlet transitions. Also, the $\Delta I_{+}$values relevant to the ac polarized exciton states have contributions from the strain perturbation of the associated macroscopic dipole interactions.

procedures used to determine the exciton-strain shifts. Recognizing this, the average exciton-strain shifts for the $b$ and ac polarized factor group components of approximately 0.6 and $0.8 \mathrm{~cm}^{-1}$, respectively, represent the most reliable values. These will be used in subsequent discussion.

The mean vibronic exciton-strain shifts listed in Table III are those which would be approximated from unpolarized spectra wherein the measu red response functions are determined by contributions from both factor group transitions. The mean strain shifts for the $(0,0),(0,1),(0,2)$, and $(0,3)$ transitions obtained from the piezoabsorption spectra are in startling agreement with the corresponding values determined from the high pressure unpolarized optical spectroscopy studies of Wiederhorn and Drickamer. ${ }^{17}$ The latter values were obtained by linear extrapolation of the transition energy shifts from the high pressure studies to a pressure of 17.6 bar which represents an average value which would be required to induce the crystal strains realized in these modulated piezoreflection experiments. The nearly perfect agreement between corresponding strain shifts obtained from the two types of spectroscopy experiments is probably fortuitous but they are certainly in the same range. However, deviations from linearity of transition energy versus pressure plots are generally minor (paper I). Hence, the linear extrapolation of the
Wiederhorn and Drickamer data to a pressure of 17.6 bar probably involves the introduction of relatively small errors into the comparison.

The strain shifts obtained from piezomodulation spectroscopy may also be compared with those values deduced from the temperature dependence of the vibronic exciton resonances. ${ }^{3}$ The $(0,0)$ vibronic exciton ener gies at $77 \mathrm{~K}$ for the $\mathrm{b}$ and ac polarized factor group components are reported to be 25096 and $25362 \mathrm{~cm}^{-1}$, respectively. ${ }^{3}$ A comparison of these energies with the corresponding room temperature value ${ }^{2}$ reveals that the exciton resonances are red shifted $214 \mathrm{~cm}^{-1}$ (for b) and $138 \mathrm{~cm}^{-1}$ (for a) due to thermal contraction of the crystal upon cooling. Estimates from the reported temperature-dependent data ${ }^{3}$ give red shifts of the $(0,0)$ resonances due to crystal cooling from room tempera ture to $77 \mathrm{~K}$ as approximately $150 \mathrm{~cm}^{-1}$ (for b) and 130 $\mathrm{cm}^{-1}$ (for a). In comparison to the thermal volume contraction $\left(3.57 \times 10^{-2}\right)$, a crystal bonded to the transducer in a piezoreflection experiment undergoes periodic volume changes of approximately $1.08 \times 10^{-4}$. The value is estimated from the known (001) planar strain and an assumed Poisson ratio of $0.3 .^{18}$ From a ratio of these two volume changes and the $(0,0)$ vibronic exciton shifts due to thermal contraction of the crystal, ${ }^{3}$ the extrapolated $(0,0)$ resonance shifts are found to be $0.45 \mathrm{~cm}^{-1}$ for $b$ and $0.39 \mathrm{~cm}^{-1}$ for a. Slightly larger strain shifts 
of $0.65 \mathrm{~cm}^{-1}$ for $b$ and $0.42 \mathrm{~cm}^{-1}$ for a are determined from a similar linear extrapolation using the thermal red shifts of $214 \mathrm{~cm}^{-1}$ for $b$ and $138 \mathrm{~cm}^{-1}$ for $a$. The foregoing suffices to demonstrate that the $(0,0)$ vibronic exciton-thermal strain shifts linearly extrapolated to small crystal strains, i.e., $1.08 \times 10^{-4}$, are comparable to those values determined from piezomodulation spectroscopy of 0.6 and $0.8 \mathrm{~cm}^{-1}$ for the $b$ and ac polarized factor group states, respectively. Hence, the comparisons vis $\dot{a}$ vis the exciton-strain sensitivities between piezomodulation spectroscopy and either the high pressure optical spectroscopy studies of Wiederhorn and Drickamer or the temperature-dependence studies of Morris and Sceats are quite favorable.

The mean strain shift for the $\hat{\mathbf{M}}_{\mathrm{A}}$ polarized transition of anthracene in the PMDA-A complex is $0.56 \mathrm{~cm}^{-1}$ (paper II). The latter is smaller than either the $b$ or ac polarized mean exciton-strain shift reported herein for pure crystalline anthracene. The comparable, albeit slightly smaller, difference in deformation potentials between the ground and excited states for the $M_{A}$ polarized transition of anthracene in PMDA-A is explicable in terms of two crystal interactions. As may be noted from Table IV, the calculated strain modulation of the exciton interaction energy for a particular vibronic transition contributes a small amount to the total shift. This contribution to the strain shift of the $\hat{\mathbf{M}}_{\mathbf{A}}$ polarized transition in PMDA - A is expected to be negligible due to the fact that exciton resonance among anthracene molecules in the crystalline complex is negligible because of the mixed stack arrangement. Fur thermore, the $(0,0)$ component of the $\hat{\mathrm{M}}_{A}$ polarized anthracene transition in PMDA-A occurs at $26100 \mathrm{~cm}^{-1}$ in both the $c_{1}$ on (010) and $E_{1}$ on (001) spectra. From the latter and the value of $27688 \mathrm{~cm}^{-1}$ for the $(0,0)$ transition energy in the vapor phase, ${ }^{19}$ one deduces a site shift of $-1588 \mathrm{~cm}^{-1}$ for the $\hat{\mathrm{M}}_{\mathbf{A}}$ polarized transition in PMDA -A. This site shift is somewhat smaller than any of the values cited for the first singlet transition in the pure crystal. Hence, that part of the strain shift arising from a modulation of the van der Waals interactions is expected to be smaller for the $\hat{M}_{A}$ polarized transition in the PMDA -A complex. One may conclude that the measured strain shifts for the $\hat{\mathrm{M}}_{\mathrm{A}}$ polarized anthracene transition in PMDA-A and in the pure crystal therefore exhibit the proper trend.

The calculated exciton strain shifts presented in Table IV should be considered. The values for $\Delta E_{ \pm}^{u, t}$ in Table IV exhibit the anticipated small differences in strain sensitivities among the vibronic components of the $1400 \mathrm{~cm}^{-1}$ progression. The average values calculated for the $b$ and ac polarized exciton-strain shifts of 0.759 and $0.748 \mathrm{~cm}^{-1}$, respectively, are in reasonable agreement with the corresponding values determined from piezomodulation spectroscopy of 0.6 and $0.8 \mathrm{~cm}^{-1}$, respectively. Although the calculated shifts take into account only $a$ and $b$ crystal strains, contributions to $\Delta E_{ \pm}^{u, r}$ from c axis strains are very small. This is a consequence of the fact that the dipole and dispersion (quadratic dipole) lattice sums converge within several molecular layers from the (001) crystal surface. Hence, it is reasonable to expect that the exciton shifts deduced from piezomodulation experiments, wherein a (001) planar is otropic strain is employed, would be in good agreement with the calculated values. The agreement between calculated and measured shifts is also aided by the absence of significant vibronic coupling. The experimental determination, although attributed to the $1400 \mathrm{~cm}^{-1}$ phonon, actually includes the strain response of the $390 \mathrm{~cm}^{-1}$ phonon. The calculation neglects this. Finally, the isotropic modulation must introduce shear, and the assumptions of the theoretical model ignore the rotations of the molecules implied by this. The isotropic strain will not affect the twofold symmetry axis but changes in the ac plane may not be negligible and this may further account for deviations observed for this factor group state.

\section{CONCLUSIONS}

Piezomodulation spectroscopy has been shown to be extremely effective in the enhancement of spectral structure. The piezoreflection spectra of the first singlet system of anthracene are observed as slightly perturbed logarithmic frequency derivatives of the direct reflection spectra. This is, to a significant degree, in contrast to the piezomodulation spectra of the oriented gas system.of the pi complex of PMDA-A where the agreement with the logarithmic derivative was, within error, exact. This indicates that the agreement of the measured and calculated derivative may be useful in determining the extent of coupling in a crystal spectrum.

The nature of the molecular crystal spectra do not permit the traditional analysis of the modulation spectra employing Seraphin coefficients; this was also found in the case of the oriented gas system. Thus, KramersKronig transformed differential optical constants must be employed for analysis. Exciton-strain shifts obtained from these data are in agreement with those obtained from the primary data. However, where the factor group splittings are to be determined, it is imperative that they be obtained from the transformed data. In the case of anthracene, the splittings obtained are in reasonable agreement with other measurements although they are somewhat smaller. The transforms of the differential data are substantially free of the difficulties associated with those of direct reflection data.

The efficacy of piezomodulation spectroscopy in obtaining the differences in deformation potentials between ground and excited crystal states is established by comparison with very high pressure studies in the spectra of anthracene and the use of data on the effect of thermal contraction of the lattice on the crystal spectra. The piezomodulation data also present experimental evidence for coupling between the first and second singlet states as well as for the presence of the ${ }^{1} L_{b}$ transition. Unfortunately, it is not at present possible to distinguish between these.

Explanation of the deviations of the piezomodulation spectra from those of the calculated logarithmic derivative and the smaller splittings deduced from the Kramers-Kronig transformed differential spectra requires additional theoretical and experimental work. Indeed, the nature of the coupling between the first and 
second singlet systems must now be examined as a function of the stress direction and such experiments are in progress.

Piezomodulation spectroscopy is a useful addition to the techniques necessary to attacking problems in the optical properties of molecular crystals. Further study and development will be necessary to define the scope of its applicability.

\section{ACKNOWLEDGMENTS}

We are grateful to $R$. R. Chance for providing the Bridgman grown sample of anthracene. Discussions with M. R. Philpott were most helpful.

${ }^{1}$ M. R. Philpott, in Advances in Chemical Physics, edited by I. Prigogine and S. A. Rice (Academic, New York, 1973), Vol. 23, p. 227.

${ }^{2}$ L. B. Clark and M. R. Philpott, J. Chem. Phys. 53, 3790 (1970).

${ }^{3}$ G. C. Morris and M. G. Sceats, Chem. Phys. 1, 376 (1973); 3, 164 (1974).

${ }^{4}$ M. R. Philpott and J. M. Turlet, J. Chem. Phys. 64, 3852 (1976).

${ }^{5}$ P. E. Schipper, Mol. Cryst. Liq. Cryst. 28, 401 (1974).

${ }^{6}$ R. Mason, Acta Crystallogr. 17, 547 (1964).
${ }^{7}$ P. W. Bridgman, Proc. Am. Acad. Sci. 76, 9, $71(1945)$; $76,1,19(1945) ; 76,3,83(1948)$.

${ }^{8}$ G. A. Samara and H. G. Drickamer, J. Chem. Phys. 37, 474 (1962).

${ }^{9}$ S. A. Rice and J. Jortner, in Physics of Solids at High Pressures, edited by C. T. Tomizuka and R. M. Emrick (Academic, New York, 1965), p. 63.

${ }^{10}$ S. Elnahwy, M. El Hamamsy, A. C. Damask, D. E. Cax, and W. B. Daniels, J. Chem. Phys. 68, 1161 (1978).

${ }^{11}$ M. R. Philpott, J. Chem. Phys. 59, 4406 (1973).

${ }^{12}$ J. Merski and C. J. Eckhardt, Chem. Phys. Lett. 63, 174 (1979).

${ }^{13}$ M. R. Philpott, J. Chem. Phys. 47, 4437 (1967); 55, 2039 (1971).

${ }^{14}$ E. Clar, Spectrochim. Acta 4, 116 (1950).

${ }^{15}$ A. Bree and S. Katagiri, J. Mol. Spectrosc. 17, 24 (1965); A. Bree, S. Katagiri, and S. R. Stuart, J. Chem. Phys. 44, 1788 (1966).

${ }^{16}$ M. Cardona, "Modulation Spectroscopy," in Solid State Physics, Supplement 11, edited by H. Ehrenreich, F. Seitz, and D. Turnbull (Academic, New York, 1969).

${ }^{17}$ S. Weiderhorn and H. G. Drickamer, J. Phys. Chem. Solids 9, 330 (1959).

${ }^{18}$ A. Bondi, Physical Properties of Molecular Crystals, Liquids and Glasses (Wiley, New York, 1968).

${ }^{19}$ J. P. Byrne and I. G. Ross, Can. J. Chem. 43, 3253 (1965).

${ }^{20}$ R. Silbey, J. Jortner, and S. A. Rice, J. Chem. Phys. 42, 1515 (1965).

${ }^{21}$ D. W. Schlosser and M. R. Philpott, Chem. Phys. 49, 181 (1980). 